H. Fujimoto

Nagoya Math. J.

Vol. 37 (1970), 91-106

\title{
ON THE AUTOMORPHISM GROUP OF A HOLOMORPHIG FIBER BUNDLE OVER A COMPLEX SPACE
}

\author{
HIROTAKA FUJIMOTO
}

\section{Dedicated to Prof. K. Ono on his 60th birthday}

\section{\$1. Introduction.}

In [8], A. Morimoto proved that the automorphism group of a holomorphic principal fiber bundle over a compact complex manifold has a structure of a complex Lie group with the compact-open topology. The purpose of this paper is to get similar results on the automorphism groups of more general types of locally trivial fiber spaces over complex spaces.1) We study automorphisms of a holomorphic fiber bundle over a complex space which has a complex space $Y$ as the fiber and a (not necessarily complex Lie) group $G$ of holomorphic automorphisms of $Y$ as the structure group (see Definition 3. 1).

The main result is the following

Theorem. For any holomorphic fiber bundle $\boldsymbol{B}$ over a complex space $X$, the automorphism group of $\boldsymbol{B}$ is a (real) Lie group if the structure group is a locally compact subgroup of the holomorphic automorphism group of the fiber and $X$ is *strongly pseudo-concave (Theorem 4.1).

As a special case of this, we see that the group of all fiber-preserving holomorphic automorphisms of a locally trivial fiber space over a *-strongly pseudo-concave complex space is a Lie group if the holomorphic automorphism group of the fiber is locally compact.

Let $\boldsymbol{B}$ be a holomorphic fiber bundle over a compact normal complex space $X$ and $M$ be an analytic set of codimension $\geqq 2$ in $X$. We can prove

Received February 5, 1969

1) In this paper, a complex space means a reduced complex analytic space which is always assumed to be $\sigma$-compact and irreducible. 
that any automorphism of the portion $\boldsymbol{B} \mid X-M$ of $\boldsymbol{B}$ over $X-M$ sufficiently near to the identity is the restriction of an automorphism of $\boldsymbol{B}$ if the structure group $G$ is a locally compact subgroup of the holomorphic automorphism group of the fiber. In this case, the automorphism group $F(B \mid X-M)$ of $\boldsymbol{B} \mid X-M$ is a Lie group. Moreover, if $G$ is a complex Lie transformation group of the fiber, $F(\boldsymbol{B} \mid X-M)$ is shown to be a complex Lie group.

\section{\$2. Holomorphic maps of complex spaces into mapping spaces.}

Let $X, Y$ and $Z$ be complex spaces. We denote the space of all holomorphic maps of $Y$ into $Z$ with the compact-open topology by $\operatorname{Hol}(Y, Z)$ and the space of all holomorphic automorphisms of $Y$ as a subspace of $\operatorname{Hol}(Y):=\operatorname{Hol}(Y, Y)$ by $\operatorname{Aut}(Y)$.

Definition 2. 1. Take an arbitrary subset $H$ of $\operatorname{Hol}(Y, Z)$. A map $g$ : $X \rightarrow H$ is called to be holomorphic if the induced map $\tilde{g}(x, y):=g(x)(y)(x \in X$, $y \in Y$ ) of $X \times Y$ into $Y$ is holomorphic (c.f. W. Kaup [5], p. 75).

By $\operatorname{Hol}(X, H)$ we denote again the space of all holomorphic maps of $X$ into $H$ with the compact-open topology, where $H$ is considered as a topological subspace of $\operatorname{Hol}(Y, Z)$. Obviously, we may consider $\operatorname{Hol}(X, \operatorname{Hol}(Y, Z))=\operatorname{Hol}(X \times Y, Z)$.

Let $h: X^{\prime} \rightarrow X$ be a holomorphic map for another complex space $X^{\prime}$. For any $g \in \operatorname{Hol}(X, H)(H \subset \operatorname{Hol}(Y, Z))$, the composite $g \cdot h: X^{\prime} \rightarrow H$ is also holomorphic. Particularly, the normalization $\mu: X^{*} \rightarrow X$ of $X$ gives the map $\mu^{*}: \operatorname{Hol}(X, H) \rightarrow \operatorname{Hol}\left(X^{*}, H\right)$ defined as $\mu^{*}(g)=g \cdot \mu$ for each $g \in \operatorname{Hol}(X, H)$.

(2. 2) The topological space $\operatorname{Hol}(X, H)$ can be canonically identified with a closed subspace of $\mathrm{Hol}\left(X^{*}, H\right)$.

The injectivity of $\mu^{*}$ is evident. While, $\operatorname{Hol}(X \times Y, Z)$ can be identified with a closed subspace of $\operatorname{Hol}\left(X^{*} \times Y, Z\right)$ because $\mu \times 1_{Y}: X^{*} \times Y \rightarrow X \times Y$ is a proper, nowhere degenerate surjective map, where $1_{Y}: Y \rightarrow Y$ is the identity map. Since we may consider $\operatorname{Hol}(X, H) \subset \operatorname{Hol}(X \times Y, Z)$ and $\operatorname{Hol}\left(X^{*}, H\right) \subset$ $\mathrm{Hol}\left(X^{*} \times Y, Z\right)$, we conclude easily the assertion (2. 2).

Each $g \in \operatorname{Hol}(X, \mathrm{Hol}(Y))$ gives a map $g^{*}:=1_{X} \times \tilde{g} \in \mathrm{Hol}(X \times Y)$ (i.e. $g^{*}(x, y)=(x, g(x) y)$ for any $\left.x \in X, y \in Y\right)$. By this correspondence, $\mathrm{Hol}(X, \mathrm{Hol}(Y))$ is homeomorphic with the subspace

$$
\operatorname{Hol}_{X}(X \times Y):=\left\{g \in \mathrm{Hol}(X \times Y) ; \pi_{X} g=\pi_{X}\right\}
$$


of $\mathrm{Hol}(X \times Y)$, where $\pi_{X}: X \times Y \rightarrow X$ is the canonical projection. Moreover, we see easily

(2. 3) Each $g^{*} \in \operatorname{Aut}(X \times Y)$ with the property $\pi_{X} g^{*}=\pi_{X}$ corresponds exactly to a map $g \in \operatorname{Hol}(X$, Aut $(Y))$ whose inverse $g^{-1}: X \rightarrow \operatorname{Aut}(Y)$ is also holomorphic, where $g^{-1}(x)=g(x)^{-1}(x \in X)$.

Let $G$ be an effective complex Lie transformation group of $Y$. We may identify $G$ with a subset of Aut $(Y)$. Since the given transforming map $\psi(g, y)=g \cdot y(g \in G, y \in Y)$ of $G \times Y$ into $Y$ is holomorphic, we have easily

(2. 4) If a map $g: X \rightarrow G$ is holomorphic with respect to the given complex structure of $G$, it is also holomorphic in the sense of Definition 2. 1.

Conversely, we can prove

Proposition 2. 5. For a normal $X$, if $g: X \rightarrow G(\subset$ Aut $(Y))$ is holomorphic in the sense of Definition 2. 1, then $g$ is holomorphic with respect to the complex structure of $G$.

To prove this, we give

Lemma 2. 6. Let $X, H, Y$ and $Z$ be complex spaces and $\varphi: H \times Y \rightarrow Z$ be a holomorphic map such that $X$ is normal and $Z$ is holomorphically separable and $\varphi(h, y)=\varphi\left(h^{\prime}, y\right)$ for any $y \in Y$ only if $h=h^{\prime}\left(h, h^{\prime} \in H\right)$. For a map $g: X \rightarrow H$, if the map $\varphi(g(x), y)(x \in X, y \in Y)$ of $X \times Y$ into $Z$ is holomorphic, then $g$ is also a holomorphic map of $X$ into $H$.

Proof of Lemma 2. 6. It suffices to show that the graph $\Gamma_{g}:=\{(x, g(x))$; $x \in X\}$ of $g$ is analytic in $X \times H$. Indeed, in this case, $\pi_{X} \mid \Gamma_{g}: \Gamma_{q} \rightarrow X$ is a bijective holomorphic map. Then $\left(\pi_{X} \mid \Gamma_{g}\right)^{-1}: X \rightarrow \Gamma_{g}$ is also holomorphic by the normality of $X$ (c.f. ([9]) and so $g=\pi_{H}\left(\pi_{X} \mid \Gamma_{g}\right)^{-1}: X \rightarrow H$ is holomorphic. Now, we consider the family $\mathscr{F}$ of all holomorphic functions $\varphi^{f, y}$ on $X \times H$ defined as

$$
\varphi^{f, y}(x, h):=f(\varphi(h, y))-f(\varphi(g(x), y)) \quad(x \in X, h \in H)
$$

for any $y \in Y$ and holomorphic function $f$ on $Z$. And we put

$$
A:=\{(x, h) \in X \times Y ; \psi(x, h)=0 \text { for any } \psi \in \mathscr{F}\} .
$$

Since $A$ is the set of the common zeros of a family of holomorphic functions on $X \times H$, it is analytic in $X \times H$ by the well-known H. Cartan's 
theorem. We want to show $\Gamma_{g}=A$. Evidently, $\Gamma_{g} \subset A$. Conversely, if $(x, h) \in A$, i.e. $f(\varphi(h, y))=f(\varphi(g(x), y))$ for any $y \in Y$ and holomorphic $f$ on $Z, \varphi(h, y)=\varphi(g(x), y)$ for any $y \in Y$ because $Z$ is holomorphically separable. By the assumption, we conclude $h=g(x)$, which shows $(x, h) \in \Gamma_{g}$. This completes the proof of Lemma 2. 6 .

Proof of Proposition 2. 5. Let $\psi: G \times Y \rightarrow Y$ be the given transforming map. We can take an open neighborhood $N$ of the identity in $G$ such that

$$
N=\left\{g \in G ; \psi(g, y) \in Y^{\prime \prime} \text { for any } y \in \bar{Y}^{\prime}\right\},
$$

where $Y^{\prime \prime}$ is a holomorphically separable open set in $Y$ and $Y^{\prime}\left(\Subset Y^{\prime \prime}\right)$ is a non-empty open set. If $\psi(g, y)=\psi\left(g^{\prime}, y\right)$ for any $y \in Y^{\prime}\left(g, g^{\prime} \in N\right)$, it remains valid for any $y \in Y$ by the theorem of identity and hence $g=g^{\prime}$ by the assumption of the effectivity. This shows that the map $\varphi:=\psi \mid N \times Y^{\prime}: N \times Y^{\prime}$ $\rightarrow Y^{\prime \prime}$ satisfies the conditions in Lemma 2.6 for the spaces $H:=N, Y:=Y^{\prime}$ and $Z:=Y^{\prime \prime}$.

Now, take a map $g: X \rightarrow G$ which is holomorphic in the sense of Definition 2. 1. Each $x_{0} \in X$ has obviously a neighborhood $U$ such that $h(x):=$ $g(x) g\left(x_{0}\right)^{-1} \in N$ for any $x \in U$. By Lemma 2.6, since $h: U \rightarrow N$ is holomorphic in the sense of Definition 2. 1, $h$ is holomorphic with respect to the complex structure of $G$. So, $g$ is also holomorphic on $U$ in the same sense.

For later uses, we give the following proposition on the continuability of holomorphic maps.

Proposition 2. 7. Let $G$ be a locally compact subgroup of $\operatorname{Aut}(Y)$. Then we can find a neighborhood $N$ of the identity in $G$ satisfying the following conditions:

For any connected open $V \subset X$ and analytically thin set $M$ in $V$ a holomorphic map $g: V-M \rightarrow G$ is holomorphically continuable to $V$ if there is an open set $D(\subset V-M)$ such that $g(D) \subset N$ and every holomorphic function on $D$ is continuable to $V$.

Proof. By the assumption, $G$ has a relatively compact neighborhood $N$ of the identity in $G$ which can be written

$$
N=\left\{g \in G ; g\left(Y_{j}^{\prime}\right) \subset Y_{j}^{\prime \prime} \text { for any } j(1 \leqq j \leqq s)\right\},
$$

where $Y_{j}^{\prime}, Y_{j}^{\prime \prime}$ are Stein open sets in $Y$ with $Y_{j}^{\prime} \Subset Y_{j}^{\prime \prime}$. We shall show that $N$ satisfies the desired conditions. Let $g: V-M \rightarrow G$ be a holomorphic map 
with $g(D) \subset N$. Since $g(x)\left(Y_{j}^{\prime}\right) \subset Y_{j}^{\prime \prime}$ for any $x \in D$, we see $\tilde{g}\left(D \times Y_{j}^{\prime}\right) \subset Y_{j}^{\prime \prime}$ for each $j(1 \leqq j \leqq s)$, where $\tilde{g}(x, y)=g(x)(y)(x \in V-M, y \in Y)$. Then $\varphi_{j}:=$ $\tilde{g} \mid D \times Y_{j}^{\prime}$ is continuable to a holomorphic map $\psi_{j}: V \times Y_{j}^{\prime} \rightarrow Y_{j}^{\prime \prime}$ by H. Kerner's theorem, because every holomorphic function on $D \times Y_{j}^{\prime}$ is continuable to $V \times Y_{j}^{\prime}$ (e.g. [1], Corollary 1 to Theorem 10, p. 63) and $Y_{j}^{\prime \prime}$ is Stein. This shows also $g(x)\left(Y_{j}^{\prime}\right) \subset Y_{j}^{\prime \prime}(1 \leqq j \leqq s)$ i.e. $g(x) \in N$ for any $x \in V-M$. Take an arbitrary $x \in M$. For any sequence $\left\{x_{n}\right\}$ in $V-M$ with $\lim _{n \rightarrow \infty} x_{n}=x,\left\{g\left(x_{n}\right)\right\}$ has a convergent subsequence in $\bar{N}$ whose limit $g_{0}(x)$ satisfies the condition $g_{0}(x)(y)=\psi_{j}(x, y)$ for any $y \in Y_{j}^{\prime}$. And any convergent subsequence of $\left\{g\left(x_{n}\right)\right\}$ has the same limit $g_{0}(x)$ by the theorem of identity. So, we obtain $g_{0}(x)=$ $\lim _{x^{\prime} \rightarrow x} g\left(x^{\prime}\right) \in \bar{N} \subset G$ for any $x \in M$. Obviously, $g_{0}$ is continuous on $V$ and so $\tilde{g}_{0}$ : $V \times Y \rightarrow Y$ is also continuous. Since $\tilde{g}_{0} l(V-M) \times Y=\tilde{g}$ is holomorphic, $\tilde{g}_{0}$ : $V \times Y \rightarrow Y$ is also holomorphic according to Riemann's theorem on removable singularities. This shows that $g$ has a holomorphic continuation $g_{0}: V \rightarrow \bar{N} \subset G$.

\section{§3. Holomorphic fiber bundles over complex spaces.}

Let $B, X$ and $Y$ be complex spaces, $\pi: B \rightarrow X$ be a holomorphic map and $G$ be a subgroup of $\operatorname{Aut}(Y)$.

Definition 3. 1. The space $B$ is said to have a structure of a holomorphic fiber bundle over $X$ with fiber $Y$ and structure group $G$ if $X$ has an open covering $\left\{U_{i} ; i \in I\right\}$ such that each $\pi^{-1}\left(U_{i}\right)(i \in I)$ is mapped onto $U_{i} \times Y$ by a biholomorphic map $\gamma_{i}$ with the property $\pi_{U_{i}} \cdot \gamma_{i}=\pi$ on $\pi^{-1}\left(U_{i}\right)$, where $\pi_{U_{i}}$ : $U_{i} \times Y \rightarrow U_{i}$ is the canonical projection, and each $\gamma_{i} \gamma_{j}^{-1} \in \operatorname{Hol}_{U_{i} \cap U_{j}}\left(\left(U_{i} \cap U_{j}\right) \times Y\right)$ $(i, j \in I)$ can be written $\gamma_{i} \gamma_{j}^{-1}(x, y)=\left(x, g_{i j}(x) y\right)\left(x \in U_{i} \cap U_{j}, y \in Y\right)$ with a holomorphic map $g_{i j}: U_{i} \cap U_{j} \rightarrow G$ which we say a transition function. Another structure on $B$ given by an open covering $\left\{V_{k}: k \in K\right\}$ and the biholomorphic maps $\gamma_{k}^{\prime}: \pi^{-1}\left(V_{k}\right) \rightarrow V_{k} \times Y$ with the property as the above is said to be equivalent to the above structure if there is a holomorphic map $\bar{g}_{k i}: V_{k} \cup U_{i} \rightarrow G$ such that $\gamma_{k}^{\prime} \gamma_{i}^{-1}(x, y)=\left(x, \bar{g}_{k i}(x) y\right) \quad\left(x \in V_{k} \cap U_{i}, y \in Y\right)$ for each $i \in I, k \in K$. As usual, a holomorphic fiber bundle is defined to be an equivalence class of structures of holomorphic fiber bundles attached to a fixed space $B$. For brevity, we denote a holomorphic fiber bundle over $X$ with fiber $Y$ and structure group $G$ by $\boldsymbol{B}=B(X, Y, G, \pi)$, or simply $\boldsymbol{B}$.

Let $\boldsymbol{B}=B(X, Y, G, \pi)$ and $\boldsymbol{B}^{\prime}=B^{\prime}\left(X^{\prime}, Y, G, \pi^{\prime}\right)$ be two holomorphic fiber bundles with the same fiber $Y$ and the same structure group $G$. 
Definition 3. 2. By a homomorphism $\varphi$ of $\boldsymbol{B}$ into $\boldsymbol{B}^{\prime}$ we mean a holomorphic map $\varphi: B \rightarrow B^{\prime}$ with the following properties;

(i) $\pi \varphi=\bar{\varphi} \pi$ for a suitable $\bar{\varphi} \in \operatorname{Hol}\left(X, X^{\prime}\right)$,

(ii) taking structures $\left\{\left(U_{i}, \gamma_{i}\right) ; i \in I\right\}$ on $B$ and $\left\{\left(V_{k}, \gamma_{k}^{\prime}\right) ; k \in K\right\}$ on $B^{\prime}$ as in Definition 3. 1, we can write $\gamma_{k}^{\prime} \gamma_{i}^{-1}=\bar{\varphi} \times \tilde{g}_{k i}$ on $\left(U_{i} \cap \bar{\varphi}^{-1}\left(V_{k}\right)\right) X Y$ with holomorphic maps $g_{k i}: U_{i} \cap \bar{\varphi}^{-1}\left(V_{k}\right) \rightarrow G(i \in I, k \in K)$, where $\tilde{g}_{k i}(x, y)=g_{k i}(x)(y)$.

If a homomorphism $\varphi: \boldsymbol{B} \rightarrow \boldsymbol{B}$ has the inverse homomorphism, it is said to be an automorphism of $\boldsymbol{B}$. By $F(\boldsymbol{B})$ we denote the set of all automorphisms of $\boldsymbol{B}$.

By definition an automorphism of $\boldsymbol{B}=B(X, Y, G, \pi)$ is a holomorphic automorphism of the space $B$. So, $F(\boldsymbol{B})$ is considered as a subspace of Aut $(B)$ with the compact-open topology. According to the well-known Bochner-Montgomery's theorem (c.f. W. Kaup [5], Satz 4, p. 83 and Satz 6 , p. 85), we see

(3. 3) If $F(\boldsymbol{B})$ is locally compact, it has a structure of a Lie transformation group of $B$.

EXAMPLE 3. 4. (i) A holomorphic principal fiber bundle over a complex space $X$ in the usual sense defines canonically a holomorphic fiber bundle over $X$ in the sense of Definition 3.1 by (2.4). If $X$ is normal, an automorphism of this bundle is nothing but an automorphism of this as a holomorphic principal fiber bundle in the usual sense (c.f. [8], p. 158) according to Proposition 2. 5.

(ii) Let $\boldsymbol{B}=B(X, Y, G, \pi)$ be assumed that $X$ is normal and $G$ is an effective complex Lie transformation group of $Y$ with the topology induced from Aut $(Y)$. In view of Proposition 2.5, $\boldsymbol{B}$ is regarded as an associated fiber bundle $\boldsymbol{P}$ over $X$ with structure group $G$ which is canonically defined by the same transition functions as $\boldsymbol{B}$. Moreover, as is easily seen, $F(\boldsymbol{B})$ is topologically isomorphic with the group of all automorphisms of $\boldsymbol{P}$.

(iii) Let $\pi: B \rightarrow X$ be a locally trivial fiber space over a complex space $X$ with fiber $Y$, i.e. $\pi$ be a holomorphic map such that, for a suitable open covering $\left\{U_{i} ; i \in I\right\}$ of $X$, each $\pi^{-1}\left(U_{i}\right)$ is biholomorphic with $U_{i} \times Y$ by a map $\gamma_{i}$ with $\pi_{U_{i}} \cdot \gamma_{i}=\pi$. Then we can define canonically a holomorphic 
fiber bundle $\boldsymbol{B}=B(X, Y$, Aut $(Y), \pi)$, where the transition functions $g_{i j}: U_{i} \cap U_{j}$ $\rightarrow \operatorname{Aut}(Y)$ are given so as to satisfy $\gamma_{i} \gamma_{j}^{-1}=1_{U_{i} \cap U_{j}} \times \tilde{g}_{i j}$. In this case, an automorphism of $\boldsymbol{B}$ means exactly a fiber-preserving holomorphic automorphism of $B$, i.e. an element $\varphi \in$ Aut $(B)$ with the property $\pi \varphi=\bar{\varphi} \pi$ for some $\bar{\varphi} \in \operatorname{Aut}(X)$.

Let $h: X^{\prime} \rightarrow X$ be a holomorphic map and $\boldsymbol{B}$ be a holomorphic fiber bundle over $X$ which is defined by the structure with the transition functions $g_{i j}$. As usual, the induced bundle $h^{-1}(\boldsymbol{B})$ over $X^{\prime}$ can be defined by the structure with the transition functions $g_{i j} \cdot h$. If $X^{\prime}$ is an open set in $X$ and $h: X^{\prime} \rightarrow X$ is the inclusion map, we call $\boldsymbol{B} \mid X^{\prime}:=h^{-1}(\boldsymbol{B})$ the portion of $\boldsymbol{B}$ over $X^{\prime}$.

Take the normalization $\mu: X^{*} \rightarrow X$ of $X$. A holomorphic fiber bundle $\boldsymbol{B}=B(X, Y, G, \pi)$ induces the bundle $\boldsymbol{B}^{*}:=\pi^{-1}(\boldsymbol{B})=B^{*}\left(X^{*}, Y, G, \pi^{*}\right)$.

Proposition 3. 5. The automorphism group $F(\boldsymbol{B})$ is topologically isomorphic with a closed subgroup of $F\left(\boldsymbol{B}^{*}\right)$.

Proof. By the definition of the induced bundle, a holomorphic map $\tilde{\mu}: B^{*} \rightarrow B$ with $\pi \cdot \tilde{\mu}=\mu \cdot \pi^{*}$ is defined canonically. And each $\varphi \in F(\boldsymbol{B})$ gives exactly one $\mu^{*}(\varphi):=\varphi^{*} \in F\left(\boldsymbol{B}^{*}\right)$ with $\tilde{\mu} \varphi^{*}=\varphi \tilde{\mu}$. The map $\mu^{*}: F(\boldsymbol{B}) \rightarrow F\left(\boldsymbol{B}^{*}\right)$ is obviously a continuous injective group homomorphism. It suffices to show the closedness of $\mu^{*}(F(\boldsymbol{B}))$ in $F\left(\boldsymbol{B}^{*}\right)$. Take a sequence $\left\{\varphi_{n}\right\}$ in $F(\boldsymbol{B})$ such that $\left\{\mu^{*}\left(\varphi_{n}\right)\right\}$ converges to $\varphi^{*}$ in $F\left(\boldsymbol{B}^{*}\right)$. Since $\tilde{\mu}$ is a proper nowhere degenerate, surjective map, we can find easily some $\varphi \in \operatorname{Hol}(B)$ with $\tilde{\mu} \varphi *=\varphi \tilde{\mu}$ and $\bar{\varphi} \in \operatorname{Hol}(X)$ with $\bar{\varphi} \pi=\pi \varphi$. Then, it can be easily proved by (2.2) that $\varphi$ satisfies the condition (ii) in Definition 3.2 in its local representation. On the other hand, $\left\{\mu^{*}\left(\varphi_{n}\right)^{-1}\right\}$ converges also to $\varphi^{*-1}$ in $F\left(\boldsymbol{B}^{*}\right)$. By the same argument as the above, we have the inverse homomorphism of $\varphi$ and so $\varphi \in F(\boldsymbol{B})$. Thus $\mu *(F(\boldsymbol{B}))$ is closed in $F\left(\boldsymbol{B}^{*}\right)$.

\section{§4. Holomorphic fiber bundles over a *-strongly pseudo- concave space.}

In this section, we prove the following main theorem.

Theorem 4. 1. For any $\boldsymbol{B}=B(X, Y, G, \pi)$, if $G$ is a locally compact subgroup of Aut $(Y)$ and $X$ is *-strongly pseudo-concave (see Definition 8. 1 in [2], p. 104), then $F(\boldsymbol{B})$ has a structure of a Lie transformation group of $B$. 
We need some preparations.

Lemma 4. 2. Let $N$ be a compact subset of Aut $(Y)$ such that $N \subset\{g \in \operatorname{Aut}(Y)$; $\left.g\left(\bar{Y}^{\prime}\right) \subset Y^{\prime \prime}\right\}$, where $Y^{\prime}$ is a non-empty open set and $Y^{\prime \prime}$ is relatively compact in some $K$-complete open subset of $Y$. Then the set

$$
\mathfrak{R}:=\{g \in \operatorname{Hol}(X, \operatorname{Aut}(Y)) ; g(x) \in N \text { for any } x \in X\}
$$

is also compact in $\mathrm{Hol}(X, \operatorname{Aut}(Y))$.

Proof. By Arzelà-Ascoli's theorem it suffices to show that $\mathfrak{N}$ is equicontinuous on $X$ with the canonical uniform structure of Aut $(Y)$ because $\{g(x) ; g \in \mathfrak{N}\}$ is included in a compact $N$ for any $x \in X$. By the assumption, the restriction map $r: N \rightarrow \operatorname{Hol}\left(Y^{\prime}, Y^{\prime \prime}\right) \quad\left(r(g):=g \mid Y^{\prime}\right.$ for each $\left.g \in N\right)$ is welldefined. With each $g \in \mathfrak{N}$ we associate the map $g^{\prime}=r \cdot g: X \rightarrow \operatorname{Hol}\left(Y^{\prime}, Y^{\prime \prime}\right)$ and $\tilde{g}^{\prime}: X \times Y^{\prime} \rightarrow Y^{\prime \prime}$ with $\tilde{g}^{\prime}(x, y)=g^{\prime}(x)(y)\left(x \in X, y \in Y^{\prime}\right)$. In view of the assumption of $Y^{\prime \prime},\left\{\tilde{g}^{\prime} ; g \in \mathfrak{N}\right\}$ is relatively compact in $\mathrm{Hol}\left(X \times Y^{\prime}, Y\right)$ ([2], Theorem 2. 1, p. 86) and so equicontinuous on $X \times Y^{\prime}$, where $Y$ is considered as a metric space with a suitable metric. Then $\left\{g^{\prime} ; g \in \mathfrak{R}\right\}\left(\subset \operatorname{Hol}\left(X, \operatorname{Hol}\left(Y^{\prime}, Y^{\prime \prime}\right)\right)\right.$ is also equicontinuous on $X$. On the other hand, since $r$ is injective by the theorem of identity, $r$ is a topological map of $N$ onto a compact subset of $\mathrm{Hol}\left(Y^{\prime}, Y^{\prime \prime}\right)$. Therefore, $\mathfrak{R}$ itself is equicontinuous.

For the proof of Tehrorem 4.1, we have only to show that $F(\boldsymbol{B})$ is locally compact by (3.3). Moreover, $X$ may be assumed to be normal. Indeed, in Theorem 4. 1, the normalization $X^{*}$ of $X$ is also *-strongly pseudo-concave and hence the induced bundle $\boldsymbol{B}^{*}$ of $\boldsymbol{B}$ over $X^{*}$ satisfies all conditions in Theorem 4. 1. If $F\left(\boldsymbol{B}^{*}\right)$ is shown to be locally compact, $F(\boldsymbol{B})$ is also locally compact according to Proposition 3. 5. In the following, $\boldsymbol{B}=B(X, Y, G, \pi)$ denotes a holomorphic fiber bundle over a normal $*$-strongly pseudo-concave space $X$ with a locally compact $G(\subset \operatorname{Aut}(Y))$.

By definition, there is a positive real-valued continuous function $v$ on $X$ such that $v$ is $*$-strongly $(\operatorname{dim} X-1)$-convex on $X-K$ (see [2], p. 101) for a suitable compact $K \subset X$ and $\{x ; v(x)>c\} \Subset X$ for any $c>0$. We put $c_{0}:=\min \{v(x) ; x \in K\}, \quad X_{c}=\{x \in X ; v(x)>c\}$ and $B_{c}:=\pi^{-1}\left(X_{c}\right)$ for any $c\left(0<c<c_{0}\right)$.

Lemma 4. 3. In the above situation, we can find a neighborhood $\boldsymbol{N}$ of the identity in $F(\boldsymbol{B})$ such that, for a suitable $c\left(<c_{0}\right)$, any sequence $\left\{\varphi_{n}\right\}$ in $\boldsymbol{N}$ has $a$ subsequence $\left\{\varphi_{n_{k}}\right\}$ having the following properties; 
(i) $\left\{\varphi_{n_{k}} \mid B_{c}\right\}$ converges to an injective map $\varphi$ in $\operatorname{Hol}\left(B_{c}, B\right)$,

(ii) the limit $\varphi$ is a homomorphism of $\boldsymbol{B} \mid X_{\boldsymbol{c}}$ into $\boldsymbol{B}$,

(iii) $\left\{\bar{\varphi}_{n_{k}}\right\}$ converges in Aut $(X)$, where $\bar{\varphi}$ denotes an automorphism of $X$ with $\pi \varphi=\bar{\varphi} \pi$ for each $\varphi \in F(\boldsymbol{B})$.

Proof. The compact set $\bar{X}_{c_{0}}$ has an open covering $\left\{V_{i}: 1 \leqq i \leqq k\right\}$ such that each $\boldsymbol{B} \mid V_{i}$ has a locally trivial bundle structure, i.e. there are biholomorphic maps $\gamma_{i}: \pi^{-1}\left(V_{i}\right) \rightarrow V_{i} \times Y$ with $\pi_{V_{i}} \cdot \gamma_{i}=\pi$ on $\pi^{-1}\left(V_{i}\right)$ and holomorphic maps $g_{i j}: V_{i} \cap V_{j} \rightarrow G$ with $\gamma_{i} \gamma_{j}^{-1}=1_{V_{i} \cap V_{j}} \times \tilde{g}_{i j}$ on $\left(V_{i} \cap V_{j}\right) \times Y(1 \leqq i, j \leqq k)$. Moreover we take open coverings $\left\{U_{i} ; 1 \leqq i \leqq k\right\}$ and $\left\{U_{i}^{\prime} ; 1 \leqq i \leqq k\right\}$ of $X$ with the property $U_{i} \Subset U_{i}^{\prime} \Subset V_{i}$ for each $i$. By Theorem 8. 3 in [2], p. 105, Aut $(X)$ is locally compact. There is a compact neighborhood $N$ of $1_{X}$ in Aut $(X)$ such that

$$
N \subset\left\{g \in \operatorname{Aut}(X) ; g\left(U_{i}^{\prime}\right) \subset V_{i} \text { for any } i\right\} .
$$

We consider the set

$$
N^{*}=\{\varphi \in F(\boldsymbol{B}) ; \text { the corresponding } \bar{\varphi} \in N\} .
$$

For each $\varphi \in N^{*}$, since $\bar{g}\left(U_{i}^{\prime}\right) \subset V_{i}$, the holomorphic maps $\varphi_{i}:=\gamma_{i} \cdot \varphi \cdot \gamma_{i}^{-1}$ : $U_{i}^{\prime} \times Y \rightarrow V_{i} \times Y$ are well-defined $(1 \leqq i \leqq k)$. Then we have holomorphic maps $g_{i}(\varphi): U_{i}^{\prime} \rightarrow G$ with the property $\varphi_{i}=\bar{\varphi} \times g_{i}(\tilde{\varphi})$ on $U_{i}^{\prime} \times Y$. On the other hand, there is a compact neighborhood $N^{\prime}$ of the identity in $G$ such that $N^{\prime} \subset\left\{g \in \operatorname{Aut}(Y) ; g\left(\bar{Y}^{\prime}\right) \subset Y^{\prime \prime}\right\}$ for open sets $Y^{\prime}, Y^{\prime \prime}$ with the same properties as in Lemma 4.2. We put

$$
N:=\left\{\varphi \in N^{*} ; g_{i}(\varphi)(x) \in N^{\prime} \text { for any } x \in \bar{U}_{i}(1 \leq i \leq k)\right\},
$$

which is obviously a neighborhood of $1_{B}$ in $F(\boldsymbol{B})$. We shall prove that $\boldsymbol{N}$ has the desired properties in Lemma 4.3 for $c:=\sup \left\{v(x) ; x \notin \cup_{i=1}^{k} U_{i}\right\}\left(<c_{0}\right)$.

Take an arbitrary $\left\{\varphi_{n}\right\}$ in $N$. Since $\left\{\bar{\varphi}_{n}\right\} \subset N$, a suitable subsequence $\left\{\bar{\varphi}_{n_{k}}\right\}$ converges to some $\bar{\varphi}$ in $\operatorname{Aut}(X)$. On the other hand, $\left\{g_{i}\left(\varphi_{n}\right)\right\}$ is relatively compact in $\operatorname{Hol}\left(U_{i}, G\right)$ by Lemma 4. 2. Relabeling indices suitably, we may assume that $\left\{g_{i}\left(\varphi_{n_{k}}\right)\right\}$ converges to some $g_{i}^{0}$ in $\operatorname{Hol}\left(U_{i}, G\right)$ for each $i$. Since $\operatorname{Hol}\left(U_{i} \times Y, Y\right)=\operatorname{Hol}\left(U_{i}, \operatorname{Hol}(Y)\right),\left\{\gamma_{i} \cdot \varphi_{n_{k}} \cdot \gamma_{i}^{-1}\right\}$ converges to $\varphi_{i}^{0}:=\tilde{\varphi} \times \tilde{g}_{i}^{0}$ in $\operatorname{Hol}\left(U_{i} \times Y, V_{i} \times Y\right)$. So, $\left\{\varphi_{n_{k}} \mid \pi^{-1}\left(U_{i}\right)\right\}$ converges to $\gamma_{i}^{-1} \cdot \varphi_{i}^{0} \cdot \gamma_{i}$ in $\mathrm{Hol}\left(\pi^{-1}\left(U_{i}\right), B\right)$. The subsequence $\left\{\varphi_{n_{k}}\right\}$ of $\left\{\varphi_{n}\right\}$ converges obviously on $B_{c} \subset U_{i} \pi^{-1}\left(U_{i}\right)$ and satisfies all desired conditions. 
Remark. If $X$ is compact, we may take $X_{c}=X$. In this case, Theorem 4.1 is an immediate consequence of Lemma 4.3.

Lemma 4. 4. Let $\left\{\varphi_{n}\right\}$ be a sequence in $F(\boldsymbol{B})$ such that $\left\{\varphi_{n} \mid B_{c}\right\}$ converges to an injective map $\varphi$ in $\operatorname{Hol}\left(B_{c}, B\right)$ for some $c<c_{0}$ and the corresponding $\left\{\bar{\varphi}_{n}\right\}$ converges to some $\bar{\varphi}$ in Aut $(X)$. Then any $x$ with $v(x)=c$ has a neighborhood $U$ such that $\left\{\varphi_{n} \mid \pi^{-1}(U)\right\}$ converges to an injective map $\varphi^{*}$ in $\operatorname{Hol}\left(\pi^{-1}(U), B\right)$.

Proof. Take a Stein neighborhood $V$ of $x$ such that $\pi^{-1}(V)$ is biholomorphic with $V \times Y$ by a map $r$. By Lemma 7.2 in [2], p. 101, there is a connected Stein neighborhood $U$ of $x(U \subseteq V)$ such that, for a suitable open $D \Subset X_{c}$, every holomorphic function on $D$ is uniquely continuable to $U$ and any 1-codimensional analytic subset of $U$ intersects $D$. Moreover, we choose Stein open sets $U^{\prime}$ and $U^{*}$ in $X$ such that $x \in U \Subset U^{\prime} \Subset V, \varphi\left(\bar{U}^{\prime}\right) \subset U^{*}, \bar{\varphi}_{n}\left(\bar{U}^{\prime}\right) \subset$ $U^{*}$ for almost all $n$ and $\pi^{-1}\left(U^{*}\right)$ is biholomorphic with $U^{*} \times Y$ by a map $\gamma^{\prime}$. Then we can define the maps $\varphi^{\prime}=\gamma^{\prime} \cdot \varphi \cdot \gamma^{-1}: U_{c}^{\prime} \times Y \rightarrow U^{*} \times Y$ and $\varphi_{n}^{\prime}:=\gamma^{\prime}$. $\varphi_{n} \cdot \gamma^{-1}: U^{\prime} \times Y \rightarrow U^{*} \times Y$ for almost all $n$, where $U_{c}^{\prime}=U^{\prime} \cap\{v>c\}$. Take an open set $D^{\prime}$ with $D \Subset D^{\prime} \Subset U_{c}^{\prime}$ and Stein neighborhoods $W, W^{\prime}$ of $y$ with $W \Subset W^{\prime} \Subset Y$ for each $y \in Y$. Since $D \times W \Subset D^{\prime} \times W^{\prime} \subset U_{c}^{\prime} \times W^{\prime}$ and $\varphi^{\prime}$ is injective on $U_{c}^{\prime} \times Y$, there is a suitable $n_{0}$ such that

$$
\varphi_{n}^{\prime}(D \times W) \Subset \varphi_{n_{0}}\left(D^{\prime} \times W^{\prime}\right) \subset \varphi_{n_{0}}^{\prime}\left(U^{\prime} \times W^{\prime}\right) \subset U^{*} \times Y
$$

for any $n \geqq n_{0}$ by Lemma 3.2 in [2], p. 89. Now, every holomorphic function on $D \times W$ is uniquely continuable to $U \times W$ (e.g. Corollary 1 in [1], p. 63) and $\varphi_{n_{0}}^{\prime}\left(U^{\prime} \times W^{\prime}\right)$ is Stein. As in the proof of Lemma 7. 3 in [2], p. 102 , we have $\varphi_{n}^{\prime}(U \times W) \subset \varphi_{n_{0}}^{\prime}\left(U^{\prime} \times W^{\prime}\right)$ for any $n \geqq n_{0}$ by $\mathrm{H}$. Kerner's theorem ([6]). Since $\varphi_{n_{0}}^{\prime}\left(U^{\prime} \times W^{\prime}\right)$ may be assumed to be relatively compact in some $K$-complete subset of $U^{*} \times Y,\left\{\varphi_{n}^{\prime} ; n \geqq n_{0}\right\}$ may be considered as a normal family in $\mathrm{Hol}\left(U \times W, U^{*} \times Y\right)$ by Theorem 2.1 in [2], p. 86. A suitable subsequence of $\left\{\varphi_{n}^{\prime}\right\}$ converges on $U \times W$. Covering $Y$ by countably many open sets $W^{\prime} s$ with the above property, we can choose a subsequence $\left\{\varphi_{n_{k}}\right\}$ of $\left\{\varphi_{n}^{\prime}\right\}$ which converges to a map $\psi$ in $\operatorname{Hol}\left(U \times Y, U^{*} \times Y\right)$. Then $\left\{\varphi_{n_{k}} \mid \pi^{-1}(U)\right\}$ converges to the map $\varphi^{*}:=\gamma^{\prime-1} \psi r$ in $\mathrm{Hol}\left(\pi^{-1}(U), B\right)$. Moreover, any subsequence of $\left\{\varphi_{n}\right\}$ has a subsequence which converges to the same limit $\varphi^{*}$. So, $\left\{\varphi_{n}\right\}$ itself converges to $\varphi^{*}$ in $\operatorname{Hol}\left(\pi^{-1}(U), B\right)$. It remains to prove the injectivity of $\varphi^{*}$ on $\pi^{-1}(U)$, or equivalently, $\psi$ on $U \times Y$. Let $E$ be the set of 
degeneracy of $\psi$, which is analytic in $U \times Y$ by $\mathrm{R}$. Remmert's theorem ([9]). Then $E$ is of codimension $\geqq 2$. In fact, if $\operatorname{codim} E=1, E \cap\left\{y=y_{0}\right\}$ can be identified with an analytic set of codimension $\geqq 1$ at $x_{0}$ in $U$ for any $\left(x_{0}, y_{0}\right) \in E$. By the assumption of $U, E$ intersects $U_{c} \times Y$. This contradicts with the injectivity of $\varphi$. Now, we apply Lemma 7. 3 in [2], p. 102, whence $\psi$ is injective on $U \times Y$. This completes the proof.

Proof of Theorem 4. 1. Take a neighborhood $N$ of $1_{B}$ in $F(\boldsymbol{B})$ satisfying the conditions in Lemma 4. 3. We shall prove that $\boldsymbol{N}^{\prime}:=\{\varphi \in F(\boldsymbol{B}) ; \varphi$ and $\left.\varphi^{-1} \in N\right\}$ is a relatively compact neighborhood of $1_{B}$ in $F(\boldsymbol{B})$. An arbitrary $\left\{\varphi_{n}\right\}$ in $N^{\prime}$ has a subsequence $\left\{\varphi_{n_{k}}\right\}$ which satisfies the conditions (i), (ii), (iii) in Lemma 4. 3. As in the proof of Theorem 7. 5, p. 103, putting

$$
\Gamma:=\left\{c ;\left\{\varphi_{n_{k}}\right\} \text { converges to an injective map in } \operatorname{Hol}\left(B_{c}, B\right)\right\},
$$

we conclude that inf $\Gamma=0$ according to Lemma 4. 4. This shows that $\left\{\varphi_{n_{h}}\right\}$ converges to some $\varphi$ in $\operatorname{Hol}(B, B)$, which satisfies obviously $\bar{\varphi} \pi=\pi \varphi$ for $\bar{\varphi}=$ $\lim _{k \rightarrow \infty} \bar{\varphi}_{n_{k}} \in \operatorname{Aut}(X)$. Then, applying the same argument to the sequence $\left\{\varphi_{n}^{-1}\right\}$, we see easily $\varphi \in \operatorname{Aut}(B)$. It remains to show $\varphi \in F(\boldsymbol{B})$. The condition (ii) in Definition 3. 2 is of local nature. For each $x \in X$ taking sufficiently small neighborhood $U$ of $x$ and $V$ of $\bar{\varphi}(x)$, we may assume that $\pi^{-1}(U)=U \times Y$, $\pi^{-1}(V)=V \times Y, \varphi(U \times Y) \subset V \times Y$ and $\varphi_{n_{k}}(U \times Y) \subset V \times Y$ for almost all $k$. Moreover, it may be assumed that $\varphi_{n_{k}}=\bar{\varphi}_{n_{k}} \times \tilde{g}_{n_{k}}$ for suitable $g_{n_{k}} \in \operatorname{Hol}(U, G)$ by Definition 3. 2 and $\varphi=\bar{\varphi} \times \tilde{g}$ on $U \times Y$ for a suitable $g \in \operatorname{Hol}(U$, Aut $(Y))$ by Example 3. 4 (iii). Then we have $g=\lim _{k \rightarrow \infty} g_{n_{k}} \in \operatorname{Hol}(U, G)$ because $G$ is closed in Aut $(Y)$. This concludes $\varphi \in F(\boldsymbol{B})$. Theorem 4.1 is completely proved.

According to Example 3. 4 (iii), Theorem 4.1 implies

Corollary 4.5. Let $B$ be a locally trivial fiber space over a *-strongly pseudo-concave complex space with fiber $Y$. If $\operatorname{Aut}(Y)$ is locally compact, the group of all fiber-preserving holomorphic automorphisms of $B$ is a Lie group.

We have also in view of Example 8. 2 in [2], p. 104,

Corollary 4. 6. Let $X$ be a compact complex manifold and $M$ be an analytic set with emdim $M \leq \operatorname{dim} X-2$. For any $\boldsymbol{B}=B(X-M, Y, G, \pi)$, if $G$ is a locally compact subgroup of $\operatorname{Aut}(Y), F(\boldsymbol{B})$ is a Lie group.

In connection with this, for a holomorphic fiber bundle $\boldsymbol{B}$ defined over 
the total $X$ we give more precise informations on automorphisms of $\boldsymbol{B} \mid X-M$ in the next section.

\section{\$5. Holomorphic fiber bundles over a compact complex space.}

Let $\boldsymbol{B}=B(X, Y, G, \pi)$ be a holomorphic fiber bundle over a compact complex space $X$. We want to study $F(\boldsymbol{B} \mid X-M)$ for a thin analytic set $M$ in $X$. By $\boldsymbol{G}^{0}$ we denote the connected component of the identity in a topological group $\boldsymbol{G}$.

Theorem 5. 1. If $X$ is a compact normal complex space, $G$ is a locally compact subgroup of Aut $(Y)$ and $M$ is of codimension $\geqq 2$, then any element in $F(\boldsymbol{B} \mid X-M)^{0}$ is the restriction of an automorphism of $\boldsymbol{B}$ over the total $X$.

Proof. It suffices to show that there is a neighborhood $\boldsymbol{N}$ of $1_{B}$ in $F(\boldsymbol{B} \mid X-M)$ such that any $\varphi \in N$ is the restriction of some $\psi \in F(\boldsymbol{B})$, because any element in $F(\boldsymbol{B} \mid X-M)$ is represented as the product of finitely many elements in $N$. The compact set $M$ has an open covering $\left\{V_{i} ; 1 \leq i \leq k\right\}$ such that, for a suitable connected open subset $D_{i}$ of $V_{i}$ with $D_{i} \Subset X-M$, any holomorphic function on $D_{i}$ is uniquely continuable to $V_{i}$ for each $i$. If we choose sufficiently small $V_{i}(1 \leqq i \leqq k)$, it may be assumed that there is another open covering $\left\{U_{i} ; 1 \leqq i \leqq k\right\}$ of $M$ such that each $U_{i}$ is Stein, $V_{i} \Subset U_{i}$ and $\boldsymbol{B} \mid U_{i}$ has a locally trivial bundle structure, and so $\pi^{-1}\left(U_{i}\right)$ is biholomorphic with $U_{i} \times Y$ by a map $\gamma_{i}$. We consider the set

$$
N:=\left\{g \in \operatorname{Aut}(X-M) ; g\left(\bar{D}_{i}^{\prime}\right) \subset U_{i} \text { and } g^{-1}\left(\bar{D}_{i}^{\prime}\right) \subset U_{i} \text { for any } i(1 \leqq i \leqq k)\right\}
$$

where $D_{i}^{\prime}$ is an open set with $D_{i} \Subset D_{i}^{\prime} \Subset U_{i}-M$. And, as in the proof of Lemma 4. 3, we put

$$
N^{*}:=\{\varphi \in F(\boldsymbol{B} \mid X-M) ; \text { the corresponding } \bar{\varphi} \in N\} .
$$

Since $\bar{\varphi}\left(\bar{D}_{i}^{\prime}\right) \subset U_{i}$ and so $\varphi\left(\pi^{-1}\left(D_{i}^{\prime}\right)\right) \subset \pi^{-1}\left(U_{i}\right)$, each $\varphi \in N^{*}$ defines a holomorphic map $g_{i}(\varphi): D_{i}^{\prime} \rightarrow G$ such that $\varphi_{i}:=\gamma_{i} \varphi \gamma_{i}^{-1}=\bar{\varphi} \times \widetilde{g_{i}(\varphi)}$ on $D_{i}^{\prime} \times Y$. Now, taking a neighborhood $N^{\prime}$ with the property in Proposition 2.7, we put

$$
N^{\prime}=\left\{\varphi \in N^{*} ; g_{i}(\varphi)(x) \in N^{\prime} \text { for any } x \in \bar{D}_{i}, 1 \leqq i \leqq k\right\} .
$$

We shall prove that $N:=\left\{\varphi \in F(B \mid X-M) ; \varphi \in N^{\prime}\right.$ and $\left.\varphi^{-1} \in N^{\prime}\right\}$ is a desired neighborhood of $1_{B}$ in $F(\boldsymbol{B})$.

Take an arbitrary $\varphi \in N$. The corresponding $\bar{\varphi} \in$ Aut $(X-M)$ with $\pi \varphi=\bar{\varphi} \pi$ defines the map $\bar{\varphi} \mid D_{i}: D_{i} \rightarrow U_{i}$ for each $i$. Since $U_{i}$ is Stein, $\bar{\varphi} \mid D_{i}$ 
is uniquely continuable to a map $\bar{\varphi}_{i}: V_{i} \rightarrow U_{i}$ by H. Kerner's theorem ([6]). Obviously, $\bar{\varphi}_{i}=\bar{\varphi}_{j}$ on $V_{i} \cap V_{j}$ if $V_{i} \cap V_{j} \neq \phi$. Therefore $\bar{\varphi}$ has a continuation to $X$, which we denote by the same notation $\bar{\varphi}$. Since $N$ is symmetric, $\bar{\varphi}^{-1}$ has also a continuation to $X$. Thus we conclude $\bar{\varphi} \in \operatorname{Aut}(X)$.

Now, since $\bar{\varphi}\left(V_{i}\right) \subset U_{i}$ by the above argument, $\varphi\left(\pi^{-1}\left(V_{i}-M\right)\right) \subset \pi^{-1}\left(U_{i}\right)$ and so the map $\varphi_{i}: r_{i} \varphi r_{i}^{-1}:\left(V_{i}-M\right) \times Y \rightarrow U_{i} \times Y$ is well-defined for each $i$. Then we get a holomorphic map $g_{i}: V_{i}-M \rightarrow G$ with $\varphi_{i}=\bar{\varphi} \times \tilde{g}_{i}$, which satisfies $g_{i}\left(D_{i}\right) \subset N^{\prime}$. By the assumption of $N^{\prime}$, each $g_{i}$ is continuable to a holomorphic map $g_{i}^{0}: V_{i} \rightarrow G$ in view of Proposition 2. 7. We consider the map $\psi_{i}:=\bar{\varphi} \times \tilde{g}_{i}^{0}: V_{i} \times Y \rightarrow U_{i} \times Y$. The map $\phi \in \operatorname{Hol}(B)$ with $\phi=\gamma_{i}^{-1} \cdot \psi_{i} \cdot \gamma_{i}$ on $\pi^{-1}\left(V_{i}\right)$ is obviously well-defined and a continuation of $\varphi$ to the total $B$. The above proof shows also $\psi \in F(\boldsymbol{B})$. This completes the proof.

As a special case of Theorem 5. 1, we have

Corollary 5.2. Let $M$ be an analytic subset of codimension $\geqq 2$ in a normal compact complex space $X$. Then any element in Aut $(X-M)^{0}$ is the restriction of an automorphism of $X$.

In Corollary 5.2, an automorphism of $X-M$ not belonging to $\operatorname{Aut}(X-M)^{0}$ is not necessarily the restriction of an automorphism of $X$. In fact, for any integer $k$, we can construct a normal compact complex space $X$ and an analytic set $M$ in $X$ with codim $M \geqq k$ such that there is an automorphism of $X-M$ which cannot be continuable to the total $X$. To construct such a space $X$ and an analytic set $M$, take a continuous proper modification $\pi: Y_{1} \rightarrow Y_{2}$ with normal compact complex spaces $Y_{1}$ and $Y_{2}$ such that, for a suitable $M_{2}:=\left\{x_{0}\right\} \subset Y_{2}, M_{1}:=\pi^{-1}\left(M_{2}\right)$ is biholomorphic with the Riemann sphere $\boldsymbol{P}^{1}$ and $\pi \mid Y_{1}-M_{1}: Y_{1}-M_{1} \rightarrow Y_{2}-M_{2}$ is a biholomorphic map (Grauert-Remmert [3], §4, p. 292), where it can be assumed that $\operatorname{dim} Y_{1}=\operatorname{dim} Y_{2} \geqq k$ for any given $k$ and $Y_{1}$ is not biholomorphic with $Y_{2}$. We consider the complex space $X:=Y_{1} \times Y_{2}$ and the analytic set $M:=\left(Y_{1} \times M_{2}\right)$ $\cup\left(M_{1} \times Y_{2}\right)$ in $X$, which is of codimension $\geqq k$. Let $g:=\pi \mid Y_{1}-M_{1}$ and $h:=g^{-1}: Y_{2}-M_{2} \rightarrow Y_{1}-M_{1}$. The map $\bar{g}(x, y):=(h(y), g(x))\left(x \in Y_{1}-M_{1}\right.$, $\left.y \in Y_{2}-M_{2}\right)$ is an automorphism of $\left(Y_{1}-M_{1}\right) \times\left(Y_{2}-M_{2}\right)=X-M$. And it cannot be the restriction of any automorphism of $X$. For, if there is some $g^{\prime} \in \operatorname{Aut}(X)$ with $\bar{g}=g^{\prime}$ on $X-M, g$ is necessarily continuable to a biholomorphic map of $Y_{1}$ onto $Y_{2}$. This is a contradiction. 
Corollary 5. 3. Let $\boldsymbol{B}=B(X, Y, G, \pi)$ be a holomorphic fiber bundle over a compact complex space and $M$ be an analytic set with codim $M \geqq 2$. If $G$ is a locally compact subgroup of $\operatorname{Aut}(Y), F(\boldsymbol{B} \mid X-M)$ has a structure of a Lie transformation group of $B-\pi^{-1}(M)$.

Proof. Without loss of generality, we may assume that $X$ is normal by Proposition 3. 5. As a special case of Theorem 4. 1, $F(\boldsymbol{B})$ has a structure of a Lie transformation group of $B$ (c.f. Remark to the proof of Lemma 4. 3). Then the closed subgroup $\boldsymbol{G}:=\left\{\varphi \in F(\boldsymbol{B}) ; \varphi\left(\pi^{-1}(M)\right)=\pi^{-1}(M)\right\}$ of $F(\boldsymbol{B})$ is also a Lie group and can be identified with a topological subgroup of $F(\boldsymbol{B} \mid X-M)$ by the restriction map. On the other hand, in view of the proof of Theorem 5. 1, there is a neighborhood $\boldsymbol{N}$ of the identity in $F(\boldsymbol{B} \mid X-M)$ such that any $\varphi \in N$ is the restriction of some $\psi \in \boldsymbol{G}$. This shows that $F(\boldsymbol{B} \mid X-M)$ and $\boldsymbol{G}$ have a common neighborhood of the identity. Thus $F(\boldsymbol{B} \mid X-M)$ is also a Lie transformation group of $B-\pi^{-1}(M)$.

Theorem 5. 4. Assume that $\boldsymbol{B}, X$ and $M$ satisfy the conditions in Theorem 5.1 and, furthermore, $G$ is a complex Lie transformation group of $Y$. Then $F(\boldsymbol{B} \mid X-M)$ has a structure of a complex Lie transformation group of $B-\pi^{-1}(M)$.

Proof. According to Example 3. 4 (ii), $\boldsymbol{B}$ is an associated fiber bundle of the canonically defined holomorphic principal fiber bundle $\boldsymbol{P}$ over $X$ and $F(\boldsymbol{B} \mid X-M)$ is isomorphic with the automorphism group of $\boldsymbol{P} \mid X-M$. As is easily seen, if the automorphism group of $\boldsymbol{P}$ is shown to be a complex Lie transformation group, $F(\boldsymbol{B} \mid X-M)$ is also a complex Lie transformation group of $B-\pi^{-1}(M)$. From the beginning, we may assume that $\boldsymbol{B}$ itself is a holomorphic principal fiber bundle, i.e. $G$ acts on the fiber $Y=G$ as the left translations. In this case, $F(\boldsymbol{B})$ is a complex Lie transformation group of $B$. For, we know that an arbitrary infinitesimal transformation group on a complex space is locally integrable (W. Kaup [5], Satz 3, p. 82) and so it can be proved that the Lie algebra of $F(\boldsymbol{B})$ is canonically isomorphic with the Lie algebra of infinitesimal transformations $D$ on $B$ with $R_{g}^{\prime} \cdot D=D$ for any $g \in G$ by the same argument as in the proof of Proposition 1 in [8], p. 163, where $R_{g}$ denotes the right translation by $g$ acting on $B$. On the other hand, the closed subgroup $\boldsymbol{G}:=\{\varphi \in F(\boldsymbol{B})$; $\left.\varphi\left(\pi^{-1}(M)\right)=\pi^{-1}(M)\right\}$ of $F(\boldsymbol{B})$ and $F(\boldsymbol{B} \mid X-M)$ have a common neighborhood of the identity. Theorem 5. 4 is a direct result of W. Kaup [5], Korollar to Satz 2, p. 80. 
Corollary 5. 5. Let $X$ be a compact complex space and $M$ be an analytic subset of $X$. If codim $M \geqq 2$, Aut $(X-M)$ has a structure of a complex Lie transformation group of $X-M$.

Proof. Let $\mu: X^{*} \rightarrow X$ be the normalization of $X$ and put $M^{*}=\mu^{-1}(M)$. The space $X^{*}-M^{*}$ is the normalization of $X-M$. Then $\operatorname{Aut}\left(X^{*}-M^{*}\right)$ is a complex Lie transformation group of $X^{*}-M^{*}$ as a special case of Theorem 5. 4. The rest of the proof of Corollary 5.5 is due to the following Lemma.

Lemma 5. 6. Let $X$ be a complex space and $\mu: X^{*} \rightarrow X$ be the normalization of $X$. If Aut $\left(X^{*}\right)$ is a complex Lie transformation group of $X^{*}$, Aut $(X)$ is also a complex Lie transformation group of $X$ as a closed subgroup of $\operatorname{Aut}\left(X^{*}\right)$.

Proof. To each $g \in \operatorname{Aut}(X)$ corresponds exactly one $g^{*} \in \operatorname{Aut}\left(X^{*}\right)$ with $g \cdot \mu=\mu \cdot g^{*}$. By this correspondence, Aut $(X)$ is considered as a closed subgroup of Aut $\left(X^{*}\right)$ (e.g. [2], Proposition 4.2) and so a real Lie group. For our purpose, it suffices to show that any real one-parameter subgroup $\left\{g_{t}\right\}$ of Aut $(X)$ can be extended to a complex one-parameter group of transformations of $X$. The given $\left\{g_{t}\right\}$ in $\operatorname{Aut}(X)$ gives a real one-parameter group $\left\{g_{t}^{*}\right\}$ in Aut $\left(X^{*}\right)$. By the assumption, $\left\{g_{t}^{*}\right\}$ is extended to a complex oneparameter group of transformations of $X^{*}$, which we denote by the same notation $\left\{g_{t}^{*}\right\}$. For each $g_{t}^{*}$, we can define a map $g_{t}^{\prime}: X \rightarrow X$ with $\mu \cdot g_{t}^{*}=$ $g_{t}^{\prime} \cdot \mu$. In fact, if $\mu\left(x_{1}^{*}\right)=\mu\left(x_{2}^{*}\right)$ for any fixed $x_{1}^{*}, x_{2}^{*}$, the holomorphic maps $\mu \cdot g_{t}^{*}\left(x_{1}^{*}\right)$ and $\mu \cdot g_{t}^{*}\left(x_{2}^{*}\right)$ of the complex number space $\boldsymbol{C}$ into $X$ coincide with each other for any real $t$. By the theorem of identity, it remains valid for any $t \in C$. This shows that the single-valued map $g_{t}^{\prime}:=\mu g_{t}^{*} \mu^{-1}: X \rightarrow X$ is well-defined. Since $\mu$ is proper nowhere degenerate surjective, $g_{t}^{\prime}(x)(t \in \boldsymbol{C}$, $x \in X)$ is obviously continuous on $\boldsymbol{C} \times X$ and $g_{t}^{\prime} g_{s}^{\prime}=g_{t+s}^{\prime}$ for any $t, s \in \boldsymbol{C}$. And $g_{t}^{\prime}(x)$ is holomorphic in $x$ for any fixed real $t$. For any $x_{0} \in X$, take a neighborhood $W$ of $\left(0, x_{0}\right)$ in $\boldsymbol{C} \times X$ such that $g_{t}^{\prime}(x) \in V$ with a Stein open set $V$ in $X$ for any $(t, x) \in W$. Then $f\left(g_{t}^{\prime}(x)\right)$ is holomorphic on $W$ for any holomorphic function $f$ on $V$ (H. Kerner [7], Hilfssatz 4, p. 285) because $f\left(g_{t} \cdot \mu(x)\right)\left(t \in C, x \in X^{*}\right)$ is holomorphic on $C \times X^{*}$. From these fact, we conclude that $g_{t}^{\prime}(x)(t \in C, x \in X)$ is holomorphic and hence $\left\{g_{t}^{\prime}\right\}$ defines a complex one-parameter transformation group of $X$ with the property $g_{t}^{\prime}=g_{t}$ for any real $t$. This completes the proof. 


\section{REFERENCES}

[1] H. Fujimoto, Vector-valued holomorphic functions on a complex space, J. Math. Soc. Japan, 17 (1965), 52-66.

[2] F. Hujimoto, On the holomorphic automorphism groups of complex spaces, Nagoya Math. J., 33 (1968), 85-106.

[ 3 ] H. Grauert and R. Remmert, Zur Theorie der Modifikationen. I, Math. Ann., 129 (1955), 274-296.

[4] H. Grauert and R. Remmert, Komplexe Räume, Math. Ann., 136 (1958), 245-318.

[5] W. Kaup, Infinitesimale Transformationsgruppen komplexer Räume, Math. Ann., 160 (1965), 72-92.

[6] H. Kerner, Über die Fortsetzung holomorpher Abbildungen, Arch. Math., 11 (1960), 44-49.

[ 7 ] H. Kerner, Über die Automorphismengruppen kompakter komplexer Räume, Arch. Math., 11 (1960), 282-288.

[8] A. Morimoto, Sur le groupe d'automorphismes d'un espace fibré principal analytique complexe, Nagoya Math. J., 13 (1958), 157-168.

[9] R. Remmert, Holomorphe und meromorphe Abbildungen komplexer Räume, Math. Ann., 133 (1957), 328-370.

Matheme

Nagoya 